\title{
Alwyn C. Scott, a subversive character in Biological Physics
}

\author{
Robert H. Austin
}

Received: 18 December 2008 / Accepted: 18 December 2008 /

Published online: 6 February 2009

(C) Springer Science + Business Media B.V. 2009

Modern nonlinear science got started when Enrico Fermi, John Pasta, Stan Ulam, and Mary Tsingou Menzel discovered a remarkable effect about nonlinear system dynamics while exploring the dynamics of a chain of masses connected by anharmonic springs via a computer simulation (in 1952!) [1, 2]. To their great surprise, the system was not ergodic under certain initial conditions, but periodically returned to its original conditions. The Fermi-Pasta-Ulam-Tsingou discovery of what could be called nonthermalization took a while to get into the public domain, and during this time the mathematician Martin Kruskal at Princeton, with Norman Zabusky at Bell Labs, discovered that there were integrable solutions to a nonlinear differential equation called the Korteweg de Vries (KdV) equation that came out of studies of nonlinear dispersive waves [3]. These solutions had remarkably robust properties and Kruskal coined the term "soliton" to describe them, a wonderful term that immediately entered the public domain. Of course, these kinds of self-sustaining structures in anharmonic systems had been seen before, most notably in the case of John Scott Russell in 1834 who discovered what he called a "wave of translation" due to the abrupt stopping of a canal barge [4]. Solitons are particularly interesting phenomena and important to physics at many different levels because they act as "particles" with an identity as they move through an anharmonic region. An excellent introduction to solitons can be found in the book by Drazin and Johnson [5].

A remarkable surge in the interest in solitons in biology occurred in the early 1970s because of two people: Alwyn C. Scott, a powerful theoretical physicist with a strong interest in nonlinear phenomena and biology, and Alexander Davydov, an eminent theoretical solid state theorist. Davydov constructed in 1973 a fully quantum mechanical model for vibrational energy propagation down the alpha helix of a protein, but it was not a very accessible article to either find (in the Western literature) or read [6]. Al Scott became fascinated with the subject and wrote several masterful reviews of the subject $[7,8]$ and helped popularize the idea that these quantum nonlinear entities might be important in biology. Among his efforts were the unearthing of Scott Russell's original

R. H. Austin ( $\square)$

Department of Physics, Princeton University, Princeton, NJ, USA

e-mail: austin@princeton.edu 
"wave of translation" observation and the tenacious recreation of this event in spite of being a card-carrying theorist.

Yesterday, a temporary lack of concentration sprawled me on the pavement and hopefully just hurt my knee. Today, I am limping around cursing my temporary immobility, and realizing how extraordinarily brave Alwyn C. Scott was, in addition to his prodigious skills as a theoretical physicist and expositor of nonlinear science. Shortly after moving to Tucson in 1985, he was struck while riding a bike by a pick-up truck which ran a red light. He was left partially paralyzed and in constant pain the rest of his life, but this did not slow down Al Scott. I have found this wonderful picture (Fig. 1, from http://www.ma. hw.ac.uk/ chris/kruskal/scott_kr_95.jpg) of Scott, Kruskal, and Chris Eilbeck of HeriotWatt University on 12 July 1995 when they witnessed a re-creation of the Scott Russell wave of translation.

Solitons do indeed exist, of course, and are very important. Although the emphasis of this memorial issue is on the possible biological role of Davydov's soliton (see the articles by Shyamsunder Erramilli, Peter Hamm, Leonor Cruzeiro, Alan Bishop, and myself), we also included some more condensed matter articles by Peter Christiansen and Al Sievers to provide a solid background. Biological solitons remain an elusive concept, and we still do not know if indeed they play a major role in biology. We leave it to the reader to decide for him- or herself and read the sad and cautionary tale written by Luca Turin about Colin McClare who tried, as Martin Kruskal's tee-shirt says, to "Subvert the Dominant Paradigm!” Alwyn C. Scott did that in many ways.

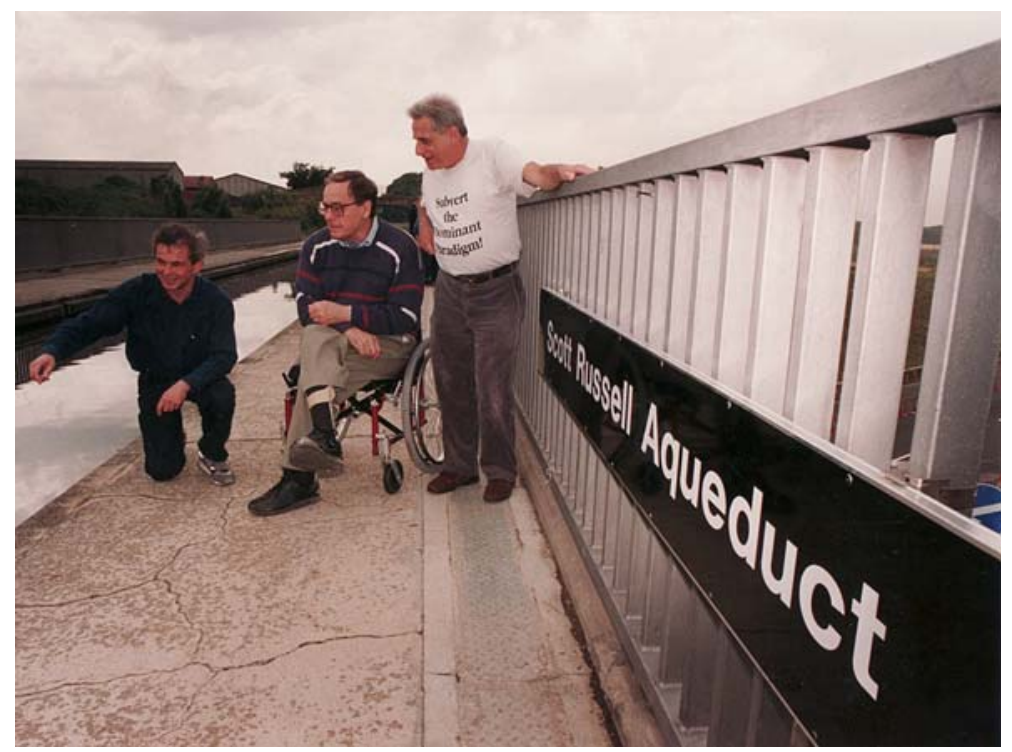

Fig. 1 Chris Eilbeck, Alwyn C. Scott, and Martin Kruskal (left to right) waiting for a Scott Russell soliton water wave (reproduced with kind permission of Chris Eilbeck, Heriot-Watt University, Edinburgh, UK) 


\section{References}

1. Fermi, E., Pasta, J., Ulam, S.: Los Alamos Scientific Laboratory Report LA-1940 (1950)

2. Dauxois, T.: Fermi, Pasta, Ulam and a mysterious lady. Phys. Today 61, 55-57 (2008)

3. Zabusky, N.J., Kruskal, M.D.: Interaction of solitons in a collisionless plasma and recurrence of initial states. Phys. Rev. Lett. 15, 240-244 (1965)

4. Russell, J.S.: Report on waves. Fourteenth meeting of the British Association for the Advancement of Science (1844)

5. Drazin, P.G., Johnson, R.S.: Solitons: An Introduction. Cambridge Texts in Applied Mathematics. Cambridge University Press, Cambridge (1989)

6. Davydov A.S.: The theory of contraction of proteins under their excitation. J. Theor. Biol. 38, 559-569 (1973)

7. Scott, A.C., Chu, F.Y.F., McLaughlin, D.W.: Soliton-A new concept in applied science. Proc. IEEE 61, $1443-1483(1973)$

8. Hyman, J.M., McLaughlin, D.W., Scott, A.C.: On Davydov's alpha-helix solitons. Physica D 3, 23-44 (1981) 